\title{
The Use of HBA1c as a Screening Test for Type 2 Diabetes and Pre-Diabetes in a Tunisian Population
}

\author{
Oueslati ${ }^{\text {I*, }}$ Belaid R, Elfaleh E, Talbi E, Khiari K, Abdelmoula J, Bouzid K and Abdallah NB \\ Université de Tunis El Manar, Faculté de Médecine de Tunis, Tunisie
}

Submission: May 05, 2017; Published: June 27, 2017

*Corresponding author: Ibtissem Oueslati, Université de Tunis El Manar, Faculté de Médecine de Tunis, hôpital Charles Nicolle, Tunisie, Email : medibtis@yahoo.fr

Abstract

Aim: To analyze the performance of HbA1c as a screening test for diabetes and pre-diabetes in a Tunisian population.

Methods: Two hundred five asymptomatic subjects with at least one risk factor for type 2 diabetes mellitus were enrolled in a crosssectional study. Oral glucose tolerance test (OGTT) and HbA1c were performed using the hexokinase method and a standardized assay by high performance liquid chromatography, respectively. Sensitivities and specificities of HbA1c for predicting diabetes and pre-diabetes compared to the diagnosis by OGTT were evaluated. A receiver operating characteristic (ROC) curve was used to determine the optimal cut-off values.

The results: Among 205 participants (mean age 43.4 \pm 9 years, sex-ratio (F/M) 3.18), 11 patients (5.4\%) had diabetes and 24 patients had pre-diabetes (11.7\%) based on OGTT. For the screening of DM using HbA1c, the area under the ROC curve was 0.95 (p < 0.001 ). With a cut-off value $\geq 6.5 \%$, HbA1c had a sensitivity of $54.5 \%$ and a specificity of $94.8 \%$. HbA1c of $5.85 \%$ gave an optimal sensitivity of $100 \%$ and specificity of $84.5 \%$ in the diagnosis of DM. For the screening of pre-diabetes using HbA1c, the area under the ROC curve was 0.665 ( $p=0.009$ ). HbA1c of $5.51 \%$ gave an optimal sensitivity of $70.8 \%$ and specificity of $63.5 \%$ in the diagnosis of pre-diabetes.

Conclusion: Based on the present findings, using the ADA cut off values in our population can be associated with a low sensitivity which translates into a missed diagnosis. Therefore, we suggest cut-off levels lower than the currently recommended values.

Keywords: Type 2 diabetes mellitus; Pre-diabetes; Screening; HbA1c; OGTT

Abbreviations: OGTT: Oral Glucose Tolerance Test; ROC: Receiver Operating Characteristic; FBG: Fasting Blood Glucose; DM: Diabetes Mellitus; ADA: American Diabetes Association; SPSS: Statistic Program For Social Sciences; AUC: Area Under The Curve

\section{Introduction}

For many years, fasting blood glucose (FBG) and oral glucose tolerance test (OGTT) have formed the basis for the screening and the diagnosis of diabetes mellitus (DM). Although FBG is a simple and a valid test, it requires patient compliance with fasting and shows intra individual and pre-analytic variabilities [1-4].

For the oral glucose tolerance test (OGTT) which is considered as the gold standard, some disadvantages could limit its use such as the necessity of a special pre test preparation by the patient including an appropriate diet for 3 days before the test and a satisfactory period of overnight fasting, its time consumption taking at least 2 hours and the intolerance of the glucose load by a significant number of people with nausea, vomiting and a delayed gastric emptying.
Thus, improved methods to detect diabetes and pre-diabetes are particularly needed such as the HbA1c testing which is in contrast with FBG and OGTT an easiest and most practical test as it is not affected by prandial status allowing measurement at any time of the day and it is only using a single blood sample $[5,6]$. Moreover, HbA1c exhibits low intra-individual variation and is relatively stable with no pre-analytic variabilities [7]. In 2010, the American Diabetes Association (ADA) proposed HbA1c $\geq 6.5 \%$ for the diagnosis of diabetes and $5.7-6.4 \%$ for impaired glucose tolerance [5]. However, the use of HbA1c as a screening test for type 2 diabetes and pre-diabetes has been widely debated in literature. Particularly, ethnic variations in HbA1c imply different cut-off values for diabetes and pre-diabetes diagnosis. The aim of the present study was to investigate the performance of $\mathrm{HbA} 1 \mathrm{c}$ as a screening test for diabetes and pre-diabetes in a Tunisian population. 


\section{Current Research in Diabetes \& Obesity Journal}

\section{Methods}

The study was performed in a cross-sectional design. Study population included non-diabetic male or female subjects with the following inclusion criteria: Age $\geq 30$ ans, presence of at least one risk factor for developing type 2 diabetes mellitus (obesity or overweight, familial history of type 2 diabetes, personal history of gestational diabetes, hypertension, dyslipidemia, personal history of cardiovascular disease...). Exclusion criteria were personal history of diabetes mellitus, personal history of chronic renal failure, anemia, hemoglobinopathies and pregnancy. After giving written informed consent, selected subjects underwent physical examination and laboratory investigations. Body height, body weight, waist circumference and blood pressure were measured. The body mass index (BMI) was calculated for each participant. Blood samples were collected for the determination of fasting plasma glucose, two hour plasma glucose levels (PG) using a standard 75g oral glucose tolerance test (OGTT), HbA1c, total cholesterol, triglycerides and HDLc levels.

Plasma glucose was measured by the hexokinase method (Architect ci8200 ; Abbott), where as HbA1c was determined in whole blood using ion-exchange high-performance liquid chromatography HPLC (D-10TM; Bio-Rad). World health organization's criteria for the diagnosis of diabetes with OGTT were used as the gold standard.

\section{Statistical analysis}

All the data were analyzed using statistic program for Social Sciences (SPSS) Program Software version 11.5. Continuous variables were expressed as mean $\pm \mathrm{SD}$, and categorical variables are expressed as percentages. The receiver operating characteristic (ROC) analysis was used to determine the sensitivity and specificity of HbA1c cut-off levels. The ROC curve draws the sensitivity against 1 minus the specificity at all possible HbA1c cut off levels. The bigger the area under the curve (AUC), the better the predictive value of HbA1c. A nominal $p$ value $<0.05$ was considered statistically significant.

\section{Results}

A total of 205 non-diabetic subjects participated in this study. Their clinical and biological features are provided in Table 1 . The mean age was $43.4 \pm 9$ years [extremes: $23-68$ ]. The majority of participants were female (76.1\%). Overweight and obesity were diagnosed in $46.8 \%$ and $25.4 \%$ of cases, respectively. OGTT revealed diabetes in 11 patients (5.4\%) and prediabetes in 24 patients (11.7\%) (Figure 1). Mean HbA1c value was $5.55 \pm 0.86$ $\%$ [extremes: 4.1-10.5].

Table 1: Clinical and biological Characteristics of the study population.

\begin{tabular}{|c|c|c|c|}
\hline & General Population $(n=205)$ & Male $(n=49)$ & Female $(n=156)$ \\
\hline Age (years) & $43.4 \pm 9$ & $46.45 \pm 9.32$ & $42.46 \pm 8.77$ \\
\hline Familial history of diabetes (\%) & 56.6 & 46.93 & 59.61 \\
\hline $\begin{array}{c}\text { Personal history of gestational } \\
\text { diabetes }(\%)\end{array}$ & 5.85 & - & 7.69 \\
\hline Weight (kg) & $73.69 \pm 11.71$ & $77.74 \pm 9.67$ & $72.40 \pm 12.03$ \\
\hline Body mass index $\left(\mathrm{kg} / \mathrm{m}^{2}\right)$ & $27.68 \pm 4.54$ & $26.24 \pm 3.29$ & $28.13 \pm 4.78$ \\
\hline Waist circumference $(\mathrm{cm})$ & $93.55 \pm 10.09$ & $95.06 \pm 8.07$ & $93.08 \pm 10.62$ \\
\hline Obesity (\%) & 25.4 & 16.3 & 28.2 \\
\hline Overweight (\%) & 46.9 & 48.9 & 46.1 \\
\hline $\begin{array}{l}\text { Systolic/diastolic blood pressure } \\
\text { (mmHg) }\end{array}$ & $122.7 \pm 13.3 / 78.2 \pm 8.9$ & $125.1 \pm 14 / 79 \pm 10$ & $122 \pm 12.9 / 77.9 \pm 8.5$ \\
\hline Hypertension (\%) & 11.2 & 14.3 & 10.3 \\
\hline Fasting blood glucose (mmol/l) & $5.2 \pm 0.78$ & $5.12 \pm 0.47$ & $5.23 \pm 0.86$ \\
\hline 2H-Glucose $(\mathrm{mmol} / \mathrm{l})$ & $6.25 \pm 2.6$ & $6.75 \pm 3.34$ & $6.09 \pm 2.32$ \\
\hline HbA1c (\%) & $5.55 \pm 0.86$ & $5.71 \pm 0.84$ & $5.23 \pm 0.86$ \\
\hline Diabetes (\%) & 5.4 & 8.2 & 4.5 \\
\hline Prediabetes (\%) & 11.7 & 22.4 & 8.3 \\
\hline Total cholesterol (mmo/l) & $4.74 \pm 0.94$ & $4.59 \pm 0.81$ & $4.79 \pm 0.97$ \\
\hline Triglycerides (mmo/l) & $1.5 \pm 1.22$ & $1.8 \pm 1.17$ & $1.41 \pm 1.22$ \\
\hline HDL-c (mmol/l) & $1.1 \pm 0.25$ & $0.98 \pm 0.23$ & $1.14 \pm 0.24$ \\
\hline LDL-c (mmol/l) & $2.97 \pm 0.76$ & $2.81 \pm 0.64$ & $3.02 \pm 0.79$ \\
\hline Dyslipidemia (\%) & 43.4 & 41.6 & 48.9 \\
\hline
\end{tabular}


Diabetes Prediabetes Normal tolerance test

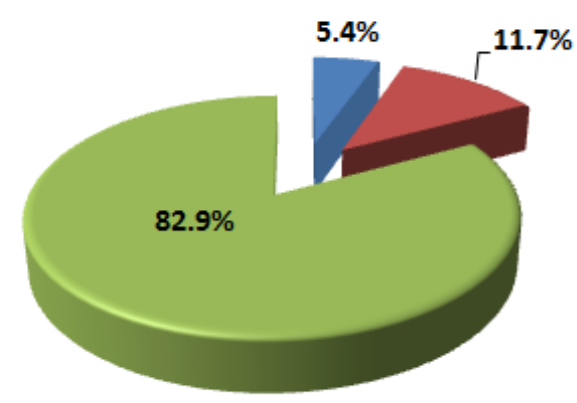

Figure 1: OGTT results.

\section{Performance of HbA1c for the screening of DM (Table}

2)

Table 2: HbA1c performances for the screening of diabetes and prediabetes.

\begin{tabular}{|c|c|c|}
\hline & $\begin{array}{c}\text { Diabetes (Cut- } \\
\text { off } \mathbf{6 . 5 \%} \text { ) }\end{array}$ & $\begin{array}{c}\text { Pre-diabetes (Cut- } \\
\text { off 5.7-6.4) }\end{array}$ \\
\hline Sensibility & $54.5 \%$ & $66.7 \%$ \\
\hline Specificity & $94.8 \%$ & $63.5 \%$ \\
\hline $\begin{array}{c}\text { Positive predictive } \\
\text { value }\end{array}$ & $37.5 \%$ & $20.5 \%$ \\
\hline $\begin{array}{c}\text { Negative predictive } \\
\text { value }\end{array}$ & $97.3 \%$ & $93.1 \%$ \\
\hline
\end{tabular}

With a cut off value of $6.5 \%$, HbA1c had a sensitivity of $54.5 \%$ and a specificity of $94.8 \%$. Figure 2 shows the ROC analysis for HbA1c in the screening of DM. The area under the curve was 0.95 [95\% confidence interval (CI): 0.914-0.988], $\mathrm{p}<0.001$. According to the ROC analysis, the cut off level for HbA1c that best predicted DM was $5.85 \%$, with a sensitivity of $100 \%$ and a specificity of $84.5 \%$. With a cut-off value of $6.05 \%$, sensibility and specificity were $81.8 \%$ and $90.2 \%$, respectively.

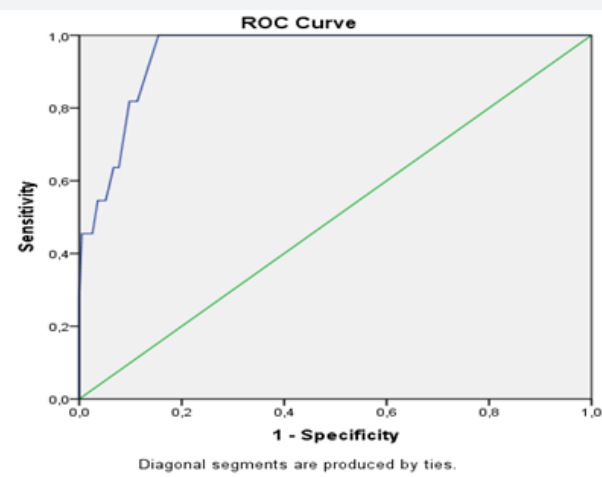

Figure 2: The ROC analysis for $\mathrm{HbA1c}$ in the screening of DM.

\section{Performance of HbA1c for the screening of pre- diabetes (Table 2)}

Figure 3 shows the ROC analysis for HbA1c in the screening of pre-diabetes. The area under the curve was 0.665 [95\% confidence interval (CI): 0.544-0.787], $\mathrm{p}<0.009$. According to the ROC analysis, the cut off level for HbA1c that best predicted pre-diabetes was $5.51 \%$, with a sensitivity of $70.8 \%$ and a specificity of $63.5 \%$.

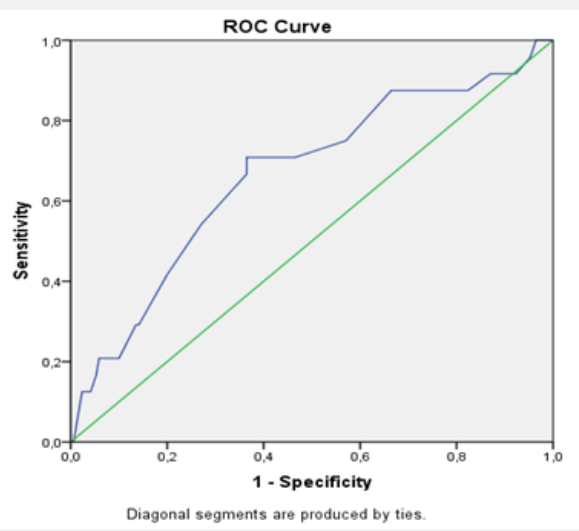

Figure 3: ROC analysis for $\mathrm{HbA} 1 \mathrm{c}$ in the screening of prediabetes.

\section{Discussion}

In this cross sectional study, HbA1c testing was a highly specific alternative to detect DM in asymptomatic subjects with at least one risk factor for diabetes. However, an HbA1c threshold value of $6.5 \%$ was associated with low sensitivity but high specificity. Several studies have evaluated the performance in terms of sensitivity and specificity of $\mathrm{HbA} 1 \mathrm{c}$ for the diagnosis of diabetes with reference to the OGTT. Their results were highly consistent with our finding.

In fact, in a recent study, Shimodaira and all had demonstrated that the sensitivity decreased, whereas the specificity increased as the cut-off levels of HbA1c increased. In diabetes, the HbA1c cut-off of $6.0 \%$ showed high sensitivity (83.7\%) and specificity (87.6\%), with a low proportion of false-negative results in disease identification [8].

In a systemic review, for a threshold value of $6.1 \%$, sensitivity ranging from 78 to $81 \%$ and specificity between 94 and $98 \%$ were observed [9]. In comparison to fasting plasma glucose (FPG), HbA1c has better specificity but lower sensitivity [9]. HbA1c values were also specifically studied in the elderly and showed a sensitivity of only $57 \%$ with a good specificity of $98 \%$ for a diagnostic threshold of $6.5 \%$ HbA1c [10]. In most reviewed study, there is an argument for population-specific cutoff points as optimum cut-offs vary by ethnic group, age, gender and population prevalence of diabetes [7].

The role of HbA1c as a predictive tool for cardiovascular diseases has been studied and it has been demonstrated that HbA1c showed superiority as compared to fasting blood glucose, but there is no threshold below which there is no risk $[11,12]$. Concerning pre-diabetes, our results revealed that $\mathrm{HbA1c}$ had lower discriminatory power compared to its utility as a diabetes screening tool. The results of the present study showed 
a modest performance of the HbA1c cut-off value of $5.7 \%$ in identifying pre-diabetes defined by OGTT-based diagnosis with low sensitivity (66.7\%) and specificity (63.5\%). These findings would indicate that a great proportion of pre-diabetic subjects would be missed during screening. Our results were similar to that reported in literature.

In fact, in a study by Mann, HbA1c cut-off value of $5.7 \%$ showed $27 \%$ sensitivity and $93 \%$ specificity in identifying prediabetes [13]. This result was confirmed by Heinaza whereas HbA1c of $5.5 \%$ gave the highest combination of specificity (76\%) and sensitivity (46\%) [14]. In a further study by Tankova, the optimal cut-off level of HbA1c for diagnosing prediabetes was $5.5 \%$ (sensitivity $71 \%$, specificity $64 \%$ ) [15].

As a diabetes and pre-diabetes screening tool, the HbA1c seems to have an imperfect sensitivity which, in clinical practice, implies a significant false-negative rate and in view of these different results, several studies suggest that the level of HbA1c should be lowered in order to have an optimal threshold with a reasonable sensitivity and specificity to detect diabetes or prediabetes at the earliest stage and thus establish an appropriate management and delay vascular complications [16].

\section{Conclusion}

Using well standardized laboratory methods, HbA1c is a reliable tool for screening DM and pre-diabetes in a high risk population. However, using the ADA cut-off values in our population can be associated with a low sensitivity which translates into a missed diagnosis. Therefore, we suggest cut-off levels lower than the currently recommended values.

\section{References}

1. Saudek CD, Herman WH, Sacks DB, Bergenstal RM, Edelman D, et al. (2008) A new look at screening and diagnosing diabetes mellitus. J Clin Endocrinol Metab 93(7): 2447-2453.

2. Gambino R (2007) Glucose: a simple molecule that is not simple to quantify. Clin Chem 53(12): 2040-2041.

3. Lin YL, Smith CH, Dietzler DN (1976) Stabilization of blood glucose by cooling with ice: an effective procedure for preservation of samples from adults and newborns. Clin Chem 22(12): 2031-2033.
4. Murphy JM, Browne RW, Hill L, Bolelli GF, Abagnato C, et al. (2000) Effects of transportation and delay in processing on the stability of nutritional and metabolic biomarkers. Nutr Cancer 37(2): 155-160.

5. American Diabetes Association (2010) Diagnosis and classification of diabetes mellitus. Diabetes Care 33(1): S62-S69.

6. Little RR, Rohlfing CL, Tennill AL, Connolly S, Hanson S (2007) Effects of sample storage conditions on glycated haemoglobin measurement: evaluation of five different high performance liquid chromatography methods. Diabetes Technol Ther 9(1): 36-42.

7. Cowie CC, Rust KF, Byrd-Holt DD, Gregg EW, Ford ES, et al. (2010) Prevalence of diabetes and high risk for diabetes using A1C criteria in the U.S. population in 1988-2006. Diabetes Care 33(3): 562-568.

8. Shimodaira M, Okaniwa S, Hanyu N, Nakayama T (2015) Optimal Hemoglobin A1c Levels for Screening of Diabetes and Prediabetes in the Japanese Population. J Diabetes Res 2015: 932057.

9. Bennett CM, Guo M, Dharmage SC (2007) HbA1c as a screening tool for detection of Type 2 diabetes: a systematic review. Diabet Med 24(4): 333343.

10. Lipska KJ, De Rekeneire N, Van Ness PH, Johnson KC, Kanaya A, et al. (2010) Identifying dysglycemic states in older adults: implications of the emerging use of hemoglobin A1c. J Clin Endocrinol Metab 95(12): 52895295 .

11. Selvin E, Steffes MW, Zhu H, Matsushita K, Wagenknecht L, et al. (2010) Glycated hemoglobin, diabetes, and cardiovascular risk in nondiabetic adults. N Engl J Med 362(9): 800811.

12. Khaw KT, Wareham N, Bingham S, Luben R, Welch A, et al. (2004) Association of hemoglobin A1c with cardiovascular disease and mortality in adults: the European prospective investigation into cancer in Norfolk. Ann Intern Med 141(6): 413-420.

13. Mann DM, Carson AP, Shimbo D (2010) Impact of A1C screening criterion on the diagnosis of pre-diabetes among U.S. adults. Diabetes Care 33(10): 2190-2195.

14. Heianza Y, Hara S, Arase Y, Saito K, Fujiwara K, et al. (2011) HbA1c 5.7$6.4 \%$ and impaired fasting plasma glucose for diagnosis of prediabetes and risk of progression to diabetes in Japan (TOPICS 3): a longitudinal cohort study. Lancet 378(9786): 147-155.

15. Tankova T, Chakarova N, Dakovska L, Atanassova I (2012) Assessment of HbA1c as a diagnostic tool in diabetes and prediabetes. Acta Diabetol 49(5): 371-378.

16. Kumar PR, Bhansali A, Ravikiran M, Bhansali S, Dutta P, et al. (2010) Utility of glycated hemoglobin in diagnosing type 2 diabetes mellitus: A Community-Based Study. J Clin Endocrinol Metab 95(6): 28322835.

\section{Your next submission with Juniper Publishers will reach you the below assets}

- Quality Editorial service

- Swift Peer Review

- Reprints availability

- E-prints Service

- Manuscript Podcast for convenient understanding

- Global attainment for your research

- Manuscript accessibility in different formats ( Pdf, E-pub, Full Text, Audio)

- Unceasing customer service

Track the below URL for one-step submission https://juniperpublishers.com/online-submission.php 